\title{
Environmental influences in schizophrenia: the known and the unknown
}

\author{
Stuart J. Leask
}

Abstract Despite much research, environmental influences that can be said to cause a schizophrenic illness remain elusive. When the effects of an (often prolonged) prodromal syndrome are taken into account, the first episode appears to come from nowhere. However, over the past couple of decades a number of factors have emerged that can be argued to influence, and not merely reflect, the illness onset. The possible effects of season and geography of birth, urbanisation, immigration, substance misuse, prenatal influenza, famine and other stresses, and obstetric complications are summarised. These varied findings, often of small effect and borderline significance, present a challenge to clinicians attempting to make sense of their patients' life experiences. Any hard conclusions still depend largely on how one formulates the illness.

This is the first article to appear the series 'Schizophrenia revisited' (see Robin McCreadie's Editorial, 2004, this issue). Future articles are planned on implementation of the NICE schizophrenia guidelines (Rowlands, 2004, in press), neuroimaging, cognitive impairment, early intervention and lifestyle issues.

The aetiology of schizophrenia remains obscure. Despite more than a century of research endeavour, we have failed to find a single factor that consistently leads either to the emergence of a schizophrenic illness, or even to a substantial increase in the risk of developing one. Although any number of environmental stresses can contribute to relapse in an individual whose schizophrenic syndrome has declared itself, that initial schizophrenic syndrome still seems to emerge from a clear blue sky. Genes undoubtedly have a part to play in the illness, but the majority of cases of schizophrenia occur in individuals with no family history of the disease. At present we have little consistent evidence to implicate any one gene, or any one environmental factor.

What has emerged is a huge research field looking at environmental exposures from myriad perspectives. Inevitably, a vast array of environmental factors have been found to be significantly associated with schizophrenia as an outcome, as schizophrenia is an illness that affects almost every aspect of life. However, what is of interest is not simply factors that are associated with having the illness, but factors that can meaningfully be thought to have something to do with its onset.
Candidate exposures are limited further by the presence of a prodromal stage of the illness that may last for months or even years, during which exposures that are apparently present before the onset of the illness proper may more correctly be seen as a consequence of this prodrome. For example, stressors such as loss of job, house or partner in the months before becoming unwell are more readily understood as consequences of the prodromal syndrome, rather than as independent contributors to the eventual illness.

Nevertheless, it is possible to identify factors that are not obviously a consequence of an individual's behaviour, that are present long before any signs of illness or prodrome, and that therefore probably can be seen as independent risk factors for a schizophrenic illness.

\section{The external environment Season of birth}

In the northern hemisphere there is reasonably consistent evidence for a $5-8 \%$ excess of births in winter and spring of children who later develop schizophrenia (Bradbury \& Miller, 1985). Initially thought to be an artefact (the earlier in a given year they are born, the older individuals will be), this finding has survived rigorous corrections (Adams \& Kendell, 1999). McGrath et al (1995) have found a similar effect for spring in the southern hemisphere.

Stuart J. Leask is a clinical lecturer at the University of Nottingham (Department of Psychiatry, Duncan Macmillan House, Porchester Road, Nottingham NG3 6AA, UK. E-mail: stuart.leask@nottingham.ac.uk) with a consultant case-load in rehabilitation psychiatry. He has worked with Professor Peter Jones in Nottingham and Professor Tim Crow in Oxford, and his research interests are centred on the developmental epidemiology of schizophrenia. 


\section{Geographical variation}

What is probably still 'the' study on geographical variations in the incidence of schizophrenia is the World Health Organization's (WHO's) ten-country study (Jablensky et al, 1992). This studied first treatment contacts and directly assessed cases in twelve centres (some urban, some rural) in Colombia, Czechoslovakia, Denmark, England, India, Ireland, Japan, Nigeria, the USA and the (then) USSR. The conclusions of this study were two-fold: that the incidence of schizophrenia as defined by ICD-9 and broadly applied Present State Examination (PSE) criteria was significantly higher in developing countries, whereas its incidence as defined purely in terms of first-rank symptoms (narrowly applied PSE criteria, the so-called 'nuclear' symptoms of schizophrenia) was constant across the world.

The conclusions one draws largely depend on how one formulates the illness. One might feel that first-rank symptoms are only a part of most clinical diagnoses, telling one nothing about prognosis, nor being exclusive to schizophrenia. Thus, many favour the notion that 'true schizophrenia' is clearly under the influence of geographically varying environmental and social factors. However, an alternative view recognises the lack of any coherent formulation of schizophrenia as a disease entity, and therefore might be more impressed by the remarkable finding, unique to schizophrenia, of a lack of variation in rate with location. Schizophrenia formulated in this way does not appear to be under the influence of any major environmental factor that varies, as almost any candidate would almost certainly vary with geography.

Subsequent studies using diagnostic systems such as ICD-10 have tended to emphasise geographical differences, and these have also supported the finding of an increased incidence in urban areas compared with rural ones. A greater incidence in developing countries has been confirmed by studies in the Caribbean, and might be felt to mark poorer social support and provision, but it should be noted that studies of course and outcome of illness suggest that these are more favourable in developing countries, despite a relative lack of drugs and other health care provision. Factors may have more to do with social cohesion than physical resources.

\section{Place of birth: urbanisation}

An increased risk of developing schizophrenia in urban compared with rural areas has been reported by many researchers since Faris \& Dunham's original 1939 study of inner-city Chicago, although initial reports suggested that this was due to increased migration of putatively prodromal

\section{Box 1 Significance and effect size}

It is important to distinguish between statistical significance and effect size. An exposure may be statistically significant (i.e. the probability of the association observed being a product of random chance is very low) but have a small effect size (i.e. the presence of the factor increases the likelihood of the observed outcome by a small amount). A small effect size, even if statistically significant, might suggest that the exposure is not related to the outcome, at least not in the fashion hypothesised.

individuals into these areas (Gerard \& Houston, 1953). More recent data suggest that the 'social drift hypothesis' might not be the whole story. Reports that greatest risk is associated with urban birth rather than later urban living (Marcelis et al, 1999) suggest that, whatever effect an urban environment may be having, it is acting early. It should be noted that studies looking at this in terms of 'deprivation' (a composite index measuring a variety of markers of social environment) have come up with some of the largest effect sizes (Box 1) in the literature (about 7 or 8$)$, but on the other hand there is a clear suggestion that the effects may not be restricted to schizophrenia, as rates of many other mental disorders are increased as well (Tarrant \& Jones, 1999). Thus, the effect may be mediated through a general increase in 'stress', making individuals more likely to express whatever illnesses they are predisposed to.

\section{Immigration}

Evidence that migrant groups have increased rates of schizophrenia dates back to 1932, when Ødergård noted this in Norwegian immigrants to the USA. Subsequent studies showed patterns that depended on the migrant group, and early on an increased rate in second-generation migrants was noted. More recently, children of Danish immigrants to Greenland have shown a relative risk of 3.7 for schizophrenia, and increased rates have been found among African-Caribbean immigrants to the UK and The Netherlands, and among African and Asian immigrants to the UK (Harrison et al, 1988; King et al, 1994; Selten \& Sijben, 1994; van Os et al, 1996; Mortensen et al, 1999).

There are many criticisms of these findings. Diagnoses from one culture might not apply in another. However, increased rates are found even in very similar cultures, for example European migrants to Western Australia. Psychiatrists from 
one culture may misdiagnose as psychosis symptoms that are acceptable in another culture. However, a UK study comparing the diagnosis of schizophrenia in African-Caribbean patients by UK and Caribbean psychiatrists concluded that, although there were disagreements at the individual level, overall rates were the same (Hickling et al, 1999). The symptom profiles and prognosis of schizophrenia in different ethnic groups do appear to differ, which might suggest that we are seeing different illnesses in the different groups, which of course could account for different rates. Estimates of the total subpopulation of immigrants might be inaccurate, as immigrants are often less rooted and less likely to be counted in census data. However, the raised rates remain in Denmark and The Netherlands, countries felt to maintain comprehensive data. Many immigrants are in an ethnic minority, and until studies report on third and later generations, teasing apart which effects derive from immigration and which from ethnic minority status is not possible.

\section{Substance misuse}

Numerous studies have described an increased rate of substance misuse in patients with schizophrenia, although frequently these have either been unrepresentative samples (e.g. Finnish army recruits) or have lacked a matched control population. An association is probably there, but whether this is a chance finding, a result of patients self-medicating, the illness (perhaps from the early prodromal stage) predisposing to substance use or vice versa remains difficult to tease out.

The exact rates of substance misuse vary with study and geography. Alcohol misuse rates in schizophrenia have been determined at $34 \%$ in the USA (Blanchard et al, 2000) and 24\% in first-episode schizophrenia in Germany (Häfner et al, 1999). The German study showed that alcohol misuse was 50\% less common in the general population (its rate was only $12 \%$ ), whereas studies from the UK are equivocal, or even find alcoholism to be more likely in the general population than in patients with schizophrenia (Bernadt \& Murray, 1986). Rates of cannabis misuse are consistently raised in schizophrenia - for example, $88 \%$ of patients in the Häfner et al study. Raised rates of the use of stimulants such as amphetamines are found in the USA, Japan and Taiwan. Opiate misuse is not associated with schizophrenia, according to studies from Germany and the USA. Finally, nicotine misuse (i.e. cigarette smoking) rates are around $90 \%$ in many studies, about three times the rates in the general population.

The question then arises of whether or not these substances can cause psychosis, and if so, whether

\section{Box 2 Influences and causes}

Philosophers have long debated the nature and definition of what makes a cause. A clear prerequisite is that a cause precedes an outcome. Causes may also be considered necessary (i.e. without this, the outcome will not occur) or sufficient (i.e. this alone is sufficient to lead to the outcome).

Since the early 20th century, science has embraced the principles of quantum mechanics, and thus we are comfortable, too, with the notion of a given exposure not inevitably causing, but merely increasing the likelihood of, a given outcome.

or not this psychosis is in any way related to schizophrenia (Box 2). Cannabis, amphetamines, cocaine and hallucinogens can all trigger brief psychotic episodes, and worsen pre-existing psychosis (for reviews see Mathers \& Ghodse (1992) on cannabis and Poole \& Brabbins (1996) on stimulants). A longitudinal study by McLellan et al (1979) of 11 individuals who misused stimulants found that 6 developed chronic psychotic states that resembled schizophrenia. Analysis by Arseneault et al (2002) of the Dunedin cohort study suggests that cannabis use as a teenager increased the risk of schizophrenia in adulthood, even after controlling for earlier 'psychotic symptoms' that pre-dated (and perhaps predisposed to) both. However, such studies are few, and there are insufficient data on rates of psychosis in regular drug users and in suitable control groups to allow conclusions as yet. The possibility of the schizophrenic prodrome predisposing to the substance misuse remains.

The temporal relationship between substance misuse and the prodromal features of schizophrenia was examined by Häfner et al (1999). Although they determined that $80 \%$ of patients were using substances before the onset of positive symptoms, this figure dropped to $27 \%$ using substances before the onset of any prodromal symptom whatsoever. They concluded that a causal relationship of some sort seemed likely, although the direction remained unclear.

Thus, the field remains uncertain. For a coherent picture to emerge we need better clarification of the timing of symptom and substance misuse onset, more care determining the type and amount of substances used, and more clarity on the symptom spectra associated with each substance. In the meantime, the clearest health need is to address the huge health and economic costs of substance misuse to this most badly off and vulnerable patient group. 


\section{The uterine environment}

The neurodevelopmental hypothesis proposes that the brains of individuals who later develop schizophrenia are subtly different from normal in ways that are apparent from an early age. Whether or not a given individual with these differences eventually develops the illness depends on whether or not they experience any number of environmental influences that then channel their ongoing neurodevelopment towards that end. Thus, there may be any number of exposures of varying effect size, none of which in and of itself is either necessary or sufficient to cause schizophrenia.

Although this theory is not new, it has risen to prominence since the 1980s, following observations that individuals who later develop schizophrenia do indeed differ from the general population in terms of their behaviour, brain structure and histopathology. Although some of the original findings have not been replicated, others have, being superseded by convincing data from longitudinal studies, imaging studies and more robust evidence from neuropathology.

\section{Prenatal influenza}

In 1988, Mednick and colleagues observed that women in the second trimester of pregnancy during the 1957-1958 influenza pandemic were twice as likely to have given birth to individuals who were later admitted with schizophrenia. There have been numerous attempts to replicate this finding. Those that succeeded (O'Callaghan et al, 1991) found effect sizes between 1.5 and 2, although many did not find any substantial effects (e.g. Kendell \& Kemp, 1989). These studies cannot rule out an effect of some related factor, such as medication or pyrexia, or the possibility that the increased rate was not present in those who were exposed. Cohort and case-control methods (which can address these issues) have also been used, and also proved negative (e.g. Crow \& Done, 1992). These latter studies might lack the power to detect small effects, but of course they do not prove that there is no association (Box 3).

Other illnesses, both pre- and perinatally, have also been implicated. A wide range of viruses that can be considered neurotrophic have been associated with central nervous system problems including learning disability, epilepsy and psychosis (Rantakallio et al, 1997). An ecological study from Finland (Suvisaari et al, 1999) found an association with polio, and a single-cohort study with robust documentation of rubella status showed an effect size for later affective psychosis of over 5 , much greater than that found for maternal influenza (Brown et al, 2000). Again, there is no evidence that allows us to establish whether these are specific effects of the infection, or effects of pyrexia, medications or the maternal immune response.

\section{Prenatal famine}

Famine in the population while a baby is in utero has been shown to be associated, in what can be argued is a dose-response manner, with later schizophrenia. Being in early gestation in The Netherlands during the Nazi blockade at the end of 1944 has an effect size of 2 for later schizophrenia (Susser \& Lin, 1992). The obvious mechanism is a relative lack of foetal nutrition, which would also explain the increased rate of schizophrenia with short birth-spacing.

\section{Prenatal stress}

Other stresses on the mother have been implicated that are not so obviously mediated to the foetus through nutrition. Birth cohort studies (e.g. Myhrman et al, 1996) have identified factors such as maternal stress (as measured by death of the spouse in the year after birth), the 'unwantedness' of the pregnancy and depression in late pregnancy as being associated with risk sizes of around 2

\section{Obstetric complications}

Related to the literature on prenatal exposure to influenza, there are many studies of the effects of obstetric complications on later rates of schizophrenia (for an overview see McNeil, 1995). A chief difficulty is that the term 'obstetric complication' can refer to almost any aspect of pre- or perinatal

\section{Box 3 No effect or no evidence?}

'No significant effect' does not equal 'evidence of no effect'. To determine that there is a $95 \%$ likelihood that two groups are the same requires around five times as many cases as would be needed to demonstrate a $95 \%$ likelihood that two groups are different. For example, if we wish to determine whether a bag of chocolate-coated peanuts is not pure, but also contains raisins, we only need to find one raisin. However, if we wish to be certain that it is pure peanuts, we would need to check all of them.

Current studies have difficulty getting enough cases to demonstrate differences; we are clearly some way off demonstrating the significant absence of an effect. 
care, and is often used to describe 'all the information that was written down at the time', rather than any specific type of perinatal insult. Nevertheless, with the huge numbers of observations made has come a rash of associations with exposures that clearly pre-date even the earliest of prodromal symptoms by a couple of decades.

Many early studies were small and of casecontrol design, often using maternal recall of the birth events, a method that has been demonstrated to be readily confounded by the mother's knowledge of the adult outcome. In a classic paper by Geddes \& Lawrie (1995), 16 case-control studies and 2 cohort studies were subjected to meta-analysis. The authors concluded that there was a small effect size (odds ratio $(\mathrm{OR})=2.0,95 \% \mathrm{CI} 1.6-2.4)$, a noticeable lack of smaller negative studies (suggesting that selection and publication biases had been at work) and big differences between the (positive) case-control findings and the (negative) cohort study findings. Impressively positive results of a large-scale casecontrol study in 1996 by Kendell et al had to be replaced by more modest, corrected findings when it was discovered that the controls in the first paper showed unrepresentatively low rates of complications (Kendell et al, 2000).

A more recent review of 12 case-control studies that had used uniform ratings for the exposures (Geddes et al, 1999) concluded that significant effects $(\mathrm{OR}=1.5-3.0)$ were present for premature rupture of membranes, premature birth (at $<37$ weeks' gestation), use of resuscitation or incubator, and a trend with respect to low birth weight $(<2.5 \mathrm{~kg})$. Birth-record data of pre-eclampsia and maternal recall of forceps delivery were also significantly associated, which suggests that birth records and maternal recall record things differently.

So, one can believe either the cohort studies, which tend to have smaller numbers of cases (and therefore limited power to detect small effect sizes), or the case-control studies, with their heterogeneity and potential for methodological flaws. Many studies have been underpowered to uncover odds ratios of 2 , given a rate of any given complication in the population of under $10 \%$. Even the meta-analysis of case-control studies (Geddes et al, 1999), with 700 cases and 935 controls, had only a $44 \%$ chance of uncovering a significant OR of 1.5. Further, one exposure may interact with another, for example rhesus incompatibility increases the risk of hypoxia during delivery, and current studies are far too underpowered to see such effects.

To address issues of interactions and power, researchers have focused on single specific hypotheses such as hypoxic/ischaemic damage, with some success (e.g. Cannon et al, 2000). But so far, different groups have used different definitions, making it
Table 1 Estimate of approximate effect sizes for preand perinatal risk factors for schizophrenia

$\begin{array}{ll}\text { Pre- or perinatal risk factor } & \begin{array}{l}\text { Approximate effect } \\ \text { size (RR or OR) }\end{array} \\ \begin{array}{ll}\text { Place or time of birth } & \\ \text { Winter birth } & 1.15 \\ \text { Urban birth } & 1.5-2.4 \\ \text { Infection } & \\ \text { Prenatal influenza } & 2 \\ \text { Prenatal respiratory infection } & 2.1 \\ \text { Prenatal rubella } & 5.2 \\ \text { Prenatal poliovirus } & 1.05 \\ \text { Neonatal and childhood } & \\ \text { CNS infection } & 4 \\ \text { Malnutrition } & \\ \text { Prenatal famine } & 2 \\ \text { Prenatal stress on mother } & \\ \text { Bereavement of spouse } & 6.2 \\ \text { Flood } & 1.8 \\ \text { 'Unwantedness' of pregancy } & 2.4 \\ \text { Maternal depression } & 1.8 \\ \text { Obstetric complications } & \\ \text { General } & 2 \\ \text { Rhesus incompatibility } & 2.8 \\ \text { Hypoxia-related } & 2.1-4.4 \\ \text { Perinatal brain damage } & 7 \\ \text { Low birth weight (<2500 g) } & 1.6 \\ \text { Pre-eclampsia } & 2.5 \\ \text { CNS, central nervous system; OR, odds ratio; RR, relative } \\ \text { risk. }\end{array} \\ \text { Source: Cannon et al, 2003: Table 5. With permission. } \\ \quad\end{array}$

difficult to draw conclusions. An alternative approach is to examine the long-term consequences of clearly identified perinatal brain damage. Although such studies have shown impressive effect sizes of 7 or more, this exposure can only account for about $5 \%$ of cases of adult schizophrenia (Jones et al, 1998). The various early risk factors and their effect sizes are summarised in Table 1.

Finally, questions are increasingly being raised about the specificity of any associations with schizophrenia. Initial reviews suggest that obstetric complications might merely be risk factors for a wide spectrum of psychiatric disorders (see Tarrant \& Jones, 1999).

\section{The nuclear environment}

It could be said that one's genes are the ultimate environmental influence, in that they affect the internal and external environment in the most profound of ways - by defining the organism. However, it is generally held that the influence of the genes is distinct from, and perhaps insulated from, any effects of the environment. 
Although my intention here is not to review the state of research into the genetic underpinnings of schizophrenia, it is important to appreciate that it is increasingly accepted that gene expression is often under the influence of environmental factors, in so-called 'gene-environment interactions'. These interactions are proposed to account for the complexity of findings, with numerous small effects from individual exposures and some complexity in genetic expression, exemplified by the monozygotic twin concordance rate for schizophrenia of under $50 \%$.

However, modelling these interactions is in itself complex, as one can propose not only a variety of ways in which environmental exposures can influence gene expression, but also that certain genetic predispositions can influence behaviour, which in turn can influence the environment, and so on. For example, IQ is in some manner under genetic control and IQ can influence behaviour to, say, alter social class, which will alter the environment and lifestyle of the individual, in turn altering the environmental exposures that may be influencing gene expression. Further, the interactions may, for any given outcome, be additive, multiplicative or independent, and these relationships may depend on the order in which they are acting.

Given the potential complexity of these interactions, it is unsurprisingly early days in terms of concluding anything at all. However, some initial attempts at modelling the heritability of schizophrenia from this perspective suggest that current estimates of genetic heritability are being exaggerated owing to an underestimation of the environmental effects (van Os \& Sham, 2003).

\section{Discussion}

The findings described above do not add up to incontrovertible evidence that schizophrenia is caused by environmental factors. Indeed, there seem to be two possible overviews of this wealth of information.

One might conclude that these numerous absent or inconsistent small effects mean that there is nothing environmental going on. This being the case, one must accept that the findings with respect to clear environmental factors are negative. Having done this, one can then focus on genetic and epigenetic models, and all that these might entail, such as evolutionary perspectives (Crow \& Done, 1993). The position is simple: a single environmental cause of large effect is not there.

Alternatively, one might conclude that these numerous small or negative effects suggest that something is going on, but that it is right on the limits of our current ability to detect it. We must look more carefully, conduct larger studies, remove confounding factors, look for 'vulnerability windows', characterise possible heterogeneity in the exposures and the outcome, and so forth. The position is simple: the situation is complex.

Although it is tempting to look back at the research data in the hope that they will suggest which viewpoint to take, the difficulty is that no study is without flaws and, given the small effect sizes and significances that are often borderline, the overall influence of these on any conclusions will remain up to the reader to decide. As yet, the data do not allow for firm conclusions independent of viewpoint.

Medical epidemiology from elsewhere might offer a valuable insight here. There are other medical outcomes for which recognised environmental effects of well-understood mechanism have been identified. Unfortunately, even for outcomes such as asthma and lung cancer, the identified exposures that have a causal role are often numerous and each is small in effect size (e.g. in asthma the effect size of air quality is less than 2); it is rare to encounter effect sizes greater than 10. A worthwhile startingpoint for any reader who wishes to examine this huge field in more detail is The Epidemiology of Schizophrenia, edited by Murray et al (2003).

In conclusion, we should be aware that, from a clinical perspective, the 'exposures' that we document in our patients' histories may well reflect a genetic predisposition to schizophrenia or be early signs of the illness prodrome. More work is needed before any of the environmental influences listed above can either be confidently rejected or considered as causal, contributing to illness onset and perhaps even worth manipulating with prevention in mind.

\section{References}

Adams, W. \& Kendell, R. E. (1999) Annual variation in birth rate of people who subsequently develop schizophrenia. British Journal of Psychiatry, 175, 522-527.

Arseneault, L., Cannon, M., Poulton, R., et al (2002) Cannabis use in adolescence and risk for adult psychosis: longitudinal prospective study. BMJ, 325, 1212-1213.

Bernadt, M. W. \& Murray, R. M. (1986) Psychiatric disorder drinking and alcoholism: what are the links? British Journal of Psychiatry, 148, 393-400.

Blanchard, J. J., Brown, S. A., Horan, W. P., et al (2000) Substance disorders in schizophrenia: review, integration, and a proposed model. Clinical Psychology Review, 20, 207234.

Bradbury, T. N. \& Miller, G. A. (1985) Season of birth in schizophrenia: a review of the evidence, methodology and etiology. Psychological Bulletin, 98, 569-594.

Brown, A. S., Schaefer, C. A. \& Wyatt, R. J. (2000) Maternal exposure to respiratory infections and adult schizophrenia spectrum disorders: a prospective birth cohort study. Schizophrenia Bulletin, 26, 287-296. 
Cannon, T. D., Rosso, I. M., Hollister, J. M., et al (2000) A prospective cohort study of genetic and perinatal influences in the etiology of schizophrenia. Schizophrenia Bulletin, 26, 351-366.

Cannon, M., Kendell, R., Susser, E., et al (2003) Prenatal and perinatal risk factors for schizophrenia. In The Epidemiology of Schizophrenia (eds R. M. Murray, P. B. Jones, E. Susser, et al), pp. 74-99. Cambridge: Cambridge University Press.

Crow, T. J. \& Done, D. J. (1992) Prenatal exposure to influenza does not cause schizophrenia. British Journal of Psychiatry, 161, 390-393.

Crow, T. J. \& Done, D. J. (1993) Sexual selection, Machiavellian intelligence and the origins of psychosis. Lancet, 342, 594-598.

Faris, R. \& Dunham, H. (1939) Mental Disorders in Urban Areas. Chicago, IL: University of Chicago Press.

Geddes, J. R. \& Lawrie, S. M. (1995) Obstetric complications in schizophrenia: a meta-analysis. British Journal of Psychiatry, 167, 786-793.

Geddes, J. R., Lawrie, S. M., Verdoux, H., et al (1999) Schizophrenia and complications of pregnancy and labor: an individual-patient data meta-analysis. Schizophrenia Bulletin, 25, 413-423.

Gerard, D. L. \& Houston, L. G. (1953) Family setting and the social ecology of schizophrenia. Psychiatric Quarterly, 27, 90-101.

Häfner, H., Maurer, K., Loffler, W., et al (1999) Onset and prodromal phase as determinants of course. In Search for the Causes of Schizophrenia: Volume IV, Balance of the Century (eds W. H. Gattaz \& H. Häfner), pp. 35-38. Berlin: Springer.

Harrison, G., Owens, D., Holten, A., et al (1988) A prospective study of severe mental disorder in AfroCaribbean patients. Psychological Medicine, 18, 643-657.

Hickling, F. W., McKenzie, K., Mullen, R., et al (1999) A Jamaican psychiatrist evaluates diagnoses at a London psychiatric hospital. British Journal of Psychiatry, 175, 283285.

Jablensky, A., Sartorius, N., Ernberg, G., et al (1992) Schizophrenia: manifestations, incidence and course in different cultures. A World Health Organization ten-country study. Psychological Medicine Monograph Supplement, 20, 1-97.

Jones, P. B., Rantakallio, P., Hartikainen, A. L., et al (1998) Schizophrenia as a long-term outcome of pregnancy, delivery and perinatal complications: a 28-year followup of the 1966 North Finland general population birth cohort. American Journal of Psychiatry, 155, 355-364.

Kendell, R. E. \& Kemp, J. W. (1989) Maternal influenza in the aetiology of schizophrenia. Archives of General Psychiatry, 46, 878-882.

Kendell, R. E., Kemp, J. W., McInneny, K., et al (2000) Obstetric complications and schizophrenia. Two case-control studies based on structured obstetric records. British Journal of Psychiatry, 176, 516-522.

King, M., Coker, E., Leavey, G., et al (1994) Incidence of psychotic illness in London: a comparison of ethnic groups. BMJ, 309, 1115-1119.

Marcelis, M., Takei, N. \& van Os, J (1999) Urbanization and risk for schizophrenia: does the effect operate before or around the time of illness onset? Psychological Medicine, 29, 1197-1203.

Mathers, D. C. \& Ghodse, A. H. (1992) Cannabis and psychotic illness. British Journal of Psychiatry, 161, 648653

McCreadie, R. G. (2004) Editorial: Schizophrenia revisited. Advances in Psychiatric Treatment, 10, 321-322.

McGrath, J., Welham, J. \& Pemberton, M. (1995) Month of birth, hemisphere of birth and schizophrenia. British Journal of Psychiatry, 167, 783-785.

McLellan, A. T., Woody, G. E. \& O'Brien, C. P. (1979) Development of psychotic illness in drug abusers: possible rôle of drug preference. New England Journal of Medicine, 301, 1310-1314

McNeil, T. F. (1995) Perinatal risk factors and schizophrenia: selective review and methodological concerns. Epidemiologic Reviews, 17, 107-112.
Mednick, S. A., Machon, R. A., Huttunen, M. O., et al (1988) Adult schizophrenia following prenatal exposure to an influenza epidemic. Archives of General Psychiatry, 45, 189-192.

Mortensen, P., Pedersen, C., Westergaard, T., et al (1999) Effects of family history and place and season of birth on the risk of schizophrenia. New England Journal of Medicine, 340, 603-608.

Murray, R. M., Jones, P. B., Susser, E., et al (eds) (2003) The Epidemiology of Schizophrenia. Cambridge: Cambridge University Press.

Myhrman, A., Rantakallio, P., Isohanni, M., et al (1996) Unwantedness of a pregnancy and schizophrenia in the child. British Journal of Psychiatry, 169, 637-640.

O'Callaghan, E., Sham, P., Takei, N., et al (1991) Schizophrenia after prenatal exposure to $1957 \mathrm{~A}_{2}$ influenza epidemic. Lancet, 337, 1248-1250.

Ødergård, O. (1932) Emigration and insanity: a study of mental disease among Norwegian born population in Minnesota. Acta Psychiatrica Neurologica Scandinavica, suppl. 4, 1-206.

Poole, R. \& Brabbins, C. (1996) Drug induced psychosis. British Journal of Psychiatry, 168, 135-138.

Rantakallio, P., Jones, P., Moring, J., et al (1997) Association between central nervous system infections during childhood and adult onset schizophrenia and other psychoses. A 28-year follow-up. International Journal of Epidemiology, 26, 837-843.

Rowlands, P. (2004) The NICE schizophrenia guidelines: the challenge of implementation. Advances in Psychiatric Treatment, 10, in press.

Selten, J. P. \& Sijben, N. (1994) First admission rate for schizophrenia in immigrants to the Netherlands. The Dutch National Register. Social Psychiatry and Psychiatric Epidemiology, 29, 71-72.

Susser, E. \& Lin, S. P. (1992) Schizophrenia after prenatal exposure to the Dutch hunger winter of 1944-1945. Archives of General Psychiatry, 49, 983-988.

Suvisaari, J., Haukka, J., Tanskanen, A., et al (1999) Association between prenatal exposure to poliovirus infection and adult schizophrenia. American Journal of Psychiatry, 156, 1100-1102.

Tarrant, J. \& Jones, P. B. (1999) Precursors to schizophrenia: do biological markers for schizophrenia have specificity? Canadian Journal of Psychiatry, 44, 335-349.

van Os, J. \& Sham, P. (2003) Gene-environment interaction in schizophrenia. In The Epidemiology of Schizophrenia (eds R. M. Murray, P. B. Jones, E. Susser, et al), p. 249. Cambridge: Cambridge University Press.

van Os, J., Castle, D. J., Takei, N., et al (1996) Psychotic illness in ethnic minorities: clarification from the 1991 census. Psychological Medicine, 26, 203-208.

\section{MCQs}

1 Studies have shown that schizophrenia:

a is more common in individuals born in the summer

b (if narrowly defined by PSE criteria) varies in incidence around the world

c carries a better prognosis in developing countries

$\mathrm{d}$ is associated with urban birth, rather than urban living

e is genuinely more common in some immigrant populations.

2 As regards studies of substance misuse and schizophrenia:

a studies have reported cannabis use rates of up to $70 \%$ in patients

$\mathrm{b}$ in the USA, alcohol misuse is not positively associated

c amphetamine misuse has been shown to cause schizophrenia 
d opiate misuse is associated with schizophrenia

e substance misuse usually pre-dates the onset of prodromal symptoms.

3 Regarding pre-natal exposure and later schizophrenia:

a prenatal influenza has consistently been found to be associated

b polio and rubella have also been associated with later psychotic illness

c stress in the pregnant mother has been associated

d a mother not wanting pregnancy has been associated

e the association between famine and later risk has shown a dose-response effect.

4 Regarding obstetric complications and later schizophrenia:

a significant associations are greater in larger studies

$b$ the effects of obstetric complications can account for the majority of cases of schizophrenia c obstetric complications clearly have effects specific to later schizophrenia

$\mathrm{d}$ maternal recall of events is a reliable information source

e low birth weight carries the highest risk.

5 Regarding environmental influences on later schizophrenia:

a childhood meningitis is associated with later schizophrenia

b although significant, the effect sizes identified are much smaller than you would expect compared with other, well-understood illnesses

c the influence of genetic inheritance on later schizophrenia is independent of the environment

d many apparent exposures could merely reflect the prodromal illness

e the effect sizes are generally greater than those for genes.

\section{Schizophrenia: full national clinical guideline on core interventions in primary and secondary care
By the National Collaborating Centre for Mental Health}

The first evidence-based clinical guideline endorsed by NICE (National Institute for Clinical Excellence) presents guidance on pharmacological, psychological and service-level interventions for schizophrenia.

In this volume, we present the complete guideline, including the evidence on which the guideline statements are based, and a detailed explanation of the methodology behind the guideline's preparation. An essential resource for all professionals involved in the management of schizophrenia, this book is a milestone in the development of truly independent and transparent clinical guidance and an essential tool in improving the quality of mental health care in the UK.

- Includes a data CD compiling all the metaanalytical data, presented both in tabular format and as Forest plots linked to each guideline statement.

2003, 264pp + 2 free CD-ROMs, A4, ISBN 190124297 8, Price $£ 50.00$

NOW AVAILABLE FROM:

Book Sales, Royal College of Psychiatrists, 17 Belgrave Square, London SW1X 8PG, UK. Tel: + 44 (0)20 72352351 ext 146. Fax: + 44 (0)20 7245 1231. Website: www.rcpsych.ac.uk

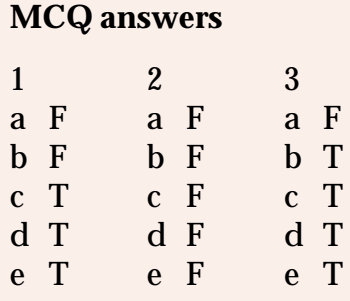

4

a F

b F

c $\mathrm{F}$

d F

e $\mathrm{F}$ 5 a $\mathrm{T}$ b F c $\mathrm{F}$ d $\mathrm{T}$ e $F$ 